\title{
Personality traits and Internet usage across generation cohorts: Insights from a nationally representative study
}

\author{
John Magnus Roos ${ }^{1,2} \cdot$ Ali Kazemi $^{1}$
}

Published online: 14 November 2018

(C) The Author(s) 2018

\begin{abstract}
Studies examining the relationship between personality and Internet usage have usually used small and non-representative samples. In the present study, we examine the relationship between the Five Factor Model of Personality and Internet usage in a large nationally representative Swedish sample $(N=1694)$. Neuroticism was negatively associated with overall Internet usage, whereas extraversion and openness to experience were shown to be positively associated with overall Internet usage. However, exploring these associations across categories of Internet usage and generation cohorts revealed some other interesting patterns. Specifically, neuroticism was negatively associated with using the Internet for activities relating to information and duties but not for leisure and social activities. Extraversion was positively associated with using the Internet for leisure and social activities among DotNets (born 1977-1999), whereas among Dutifuls (born 1910-1945) and Baby Boomers (born 1946-1964) extraversion was positively associated with using the Internet for information and duty activities. Openness to experience was positively associated with Internet usage but only among Baby Boomers. Conscientiousness was a significant predictor of Internet usage only for DotNets and GenXers (born 1965-1976). In these cohorts, conscientiousness was positively associated with using the Internet for information and duty activities but negatively associated with using the Internet for leisure and social activities. Apparently, understanding the relationship between personality and Internet usage is not possible without considering the modifying role of categories of Internet usage and generation cohorts. The implications of the results for theory and practice are discussed in detail.
\end{abstract}

Keywords Internet usage $\cdot$ Personality $\cdot$ Traits $\cdot$ Generation cohorts

\section{Introduction}

For most people in Sweden (and other wired countries), the Internet has become an essential part of everyday life. People use the Internet to search information, communicate, shop, listen to music, watch movies, play games, etc. The Internet is thus used to collect information related to work and studies, but also as a source of entertainment. As the Internet becomes

John Magnus Roos

magnus.roos@his.se

Ali Kazemi

ali.kazemi@his.se

1 School of Health and Education, University of Skövde, Höskolevägen 1, SE-541 28 Skövde, Sweden

2 Centre for Consumer Research, University of Gothenburg, PO Box 606, SE-405 30 Gothenburg, Sweden an increasingly integrated part of everyday life (Elliott and Urry 2010; Persson and Eriksson-Björling 2011), it is of crucial importance to enhance our understanding of the determinants of online human behavior. Amichai-Hamburger (2007) claims that personality is an important factor in this context. Therefore, the present study aims to investigate the extent to which personality is related to Internet activities, whether this relationship depends on the type of Internet activity and whether it differs across generation cohorts. These questions have not been fully addressed in previous research, and we believe that addressing these questions contribute to this line of research as well as helps in designing the Internet for different types of users with different needs and preferences. Moreover, as most previous studies on Internet usage and personality have used small samples and/ or samples limited to young adults, the external validity of their findings might be limited. As a remedy, we use a nationally representative sample including different age categories in this study. 
The paper is organized as follows. First, we provide a definition of Internet usage. Next, we review previous research on personality traits and internet usage, followed by a brief review of how generation cohort and gender relate to Internet usage. We then present and discuss findings from a survey study addressing the relationships between personality traits and Internet usage across four different generation cohorts controlling for age and gender.

\section{Internet Usage}

We define Internet usage in terms of overall Internet usage as well as categories of Internet usage. The Internet is an essential part of our everyday life and has grown so varied in its offerings that focusing only on overall Internet usage might fail to capture important individual differences in user activities. Therefore, we include categories of Internet usage.

"Overall Internet usage" (sometimes referred to as "global Internet usage" or "general Internet usage") is the total Internet usage regardless of the type of activity that the user is engaged in. "Categories of Internet usage" (sometimes referred to as "Usage by category" or "Factors of Internet services") pertain to different Internet activity categorizations. Thus, similar activities are clustered together. Different Internet activity categorizations have been proposed. One categorization that has been used in some studies (e.g., Landers and Lounsbury 2006; Mark and Ganzach 2014) was proposed by Hamburger and Ben-Artzi (2000) and identified three categories of Internet services, i.e., information services, social services (e.g., membership in chat groups), and leisure services (e.g., random Internet surfing, searching for pages with adult content).

\section{Personality Traits and Internet Usage}

Theories of personality conceptualize behavioral differences in terms of wide psychological characteristics (i.e., traits). Traits are partly inherited and remain relatively stable throughout the lifespan, especially after the age of 16 . Personality theorists have proposed different taxonomies of personality traits over the years. The Five Factor Model of Personality is by far the most popular descriptive taxonomy of personality traits. It posits that there are five major and universal factors of personality, i.e., neuroticism, extraversion, openness to experience, agreeableness, and conscientiousness (Costa and McCrae 1992; Digman 1990; Soto et al. 2011).

One of the widely used abbreviations of the Five Factor Model of Personality is NEOAC (i.e., Neuroticism, Extraversion, Openness to experience, Agreeableness, and Conscientiousness) (e.g., Chamorro-Premuzic 2012) and we will therefore, for the sake of clarity, in the following subsections present and discuss the personality traits in this order.

\section{Neuroticism}

Neuroticism is usually described in terms of anxiety, vulnerability, tension, distress and low self-confidence. Thus, people high in neuroticism are anxious and nervous, while people low in neuroticism (i.e., emotionally stable) tend to be more relaxed and calm (Costa and McCrae 1992). Previous studies on neuroticism and Internet usage have reported mixed results. There are studies that have shown that neuroticism is negatively associated with Internet usage and have explained it by referring to the concern for privacy and security when using the Internet among people high in neuroticism (e.g., Tuten and Bosnjak 2001). Another explanation is a missmatch between the design of the Internet (e.g., using the search engines to gather information) and the typical characteristics of people high in neuroticism (e.g., anxiety and tension). That is, searching for and gathering information takes time and requires patience and people high in neuroticism tend to be restless and lack patience.

Some other research studies have reported a positive relationship between neuroticism and Internet usage (e.g., Hamburger and Ben-Artzi 2000) and have explained it by referring to the need of people high in neuroticism to search for information on the Internet to reduce uncertainty. Another explanation states that people high in neuroticism tend to be lonely and shy, and therefore they use the Internet to express their identity (i.e., "real me") and to satisfy their need for belongingness (Amichai-Hamburger et al. 2002; Amiel and Sargent 2004). A third explanation states that the Internet provides several leisure activities, such as listening to music and watching movies, which can be especially relaxing for people who are high in neuroticism (Swickert et al. 2002). More recent research shows that people high in neuroticism are using the Internet more frequently, especially in regard to leisure and social activities such as blogs and social media (Correa et al. 2010; Guadagno et al. 2008). Recent research also shows that neuroticism is positively related to overall Internet usage (Mark and Ganzach 2014). Therefore, we expect neuroticism to be positively related to Internet activities that may be instrumental in reducing anxiety in people high in neuroticism. However, there is also reason for us to believe that people high in neuroticism might avoid engagement in some Internet activities (e.g., banking and authority services) which may cause them stress. Thus, the sign of the relationship between neuroticism and Internet usage is most likely dependent on the type of Internet activity.

\section{Extraversion}

Extraversion is usually described in terms of sociability, high level of activity, having positive emotions, impulsiveness and assertiveness. Thus, people high in extraversion are sociable, outgoing and seek stimulation from their environment, while 
people low in extraversion are more reserved and withdrawn (Costa and McCrae 1992).

Previous studies show that the Internet primarily was used by people low in extraversion (i.e., introverts) (e.g., AmichaiHamburger 2002; Amichai-Hamburger et al. 2002; Landers and Lounsbury 2006). This line of research seemed to suggest that people who were low in extraversion were attracted to the Internet because of its anonymity. Moreover, Kraut et al. (2002) proposed that the Internet compensated for people's needs of social support and therefore mostly benefited introverts and those with less social support (i.e., the social compensation model). The social compensation model posits that introverted users profit most from using the Internet, because the Internet paves the way for them to become more social. This model is in contrast to the rich-get-richer model which posits that the Internet is primarily benefitting people who already are sociable. That is, users who already have a good social life (e.g., spending time with friends and acquaintances) use the Internet to reinforce their social ties both by adding members to their existing networks and by strengthening existing ties (Kraut et al. 2002). If this reasoning holds, extraverts should benefit more from using the Internet than their more introvert counterparts who have more limited networks. More recent studies corroborate this line of reasoning and indicate a positive relationship between extraversion and Internet usage (e.g., Mark and Ganzach 2014; McElroy et al. 2007). Extraversion has also been shown to be positively related to using social media (Correa et al. 2010).

As the Internet is better designed for the extraverts' need to maintain and organize their social contacts, the fact that the Internet offers a wide range of external stimulations (e.g., music, films, games, information), and that extraverts seek stimulation form their environment, we expect extraversion to be positively associated with Internet usage.

\section{Openness to Experience}

Openness to experience is usually described in terms of behavioral flexibility, intellectual curiosity, aesthetic sensitivity, vivid imagination, unconventional attitudes, need for variety, novelty, and change. Thus, people high in openness to experience enjoy trying new things and usually have a wide range of interests, while people low in openness to experience tend to be more cautious and conservative (Costa and McCrae 1992).

Previous research studies show that openness to experience is positively related to using the Internet for random surfing, seeking entertainment and searching for specific information (Tuten and Bosnjak 2001). More recent studies indicate that people high in openness to experience are more likely to seek and test more new Internet activities, such as using blogs (Guadagno et al. 2008) and social media (Correa et al. 2010). Openness to experience seems to be positively related to both overall Internet usage and categories of Internet usage
(Kim and Jeong 2015; Mark and Ganzach 2014). In line with previous research, we expect openness to experience to be positively associated with Internet usage.

\section{Agreeableness}

Agreeableness is usually described in terms of prosocial behavior, courtesy, friendliness, flexibility, tolerance, altruism, cooperativeness, good-naturedness, and tender-mindedness. People high in agreeableness also tend to rely more on other people and forgive other people more easily than people low in agreeableness who tend to be more suspicious and argumentative (Costa and McCrae 1992). Landers and Lounsbury (2006) found a negative relationship between agreeableness and overall Internet usage. However, agreeableness was shown to be unrelated to percent time spent on the Internet by category of usage (i.e., communication, leisure, and academic). Generally speaking, agreeableness seems to be unrelated to Internet usage (Hughes et al. 2012; Kim and Jeong 2015; Mark and Ganzach 2014; McElroy et al. 2007; Tuten and Bosnjak 2001).

Agreeableness has consistently been described as a universal personality factor and as such, for the sake of completeness, it is included in the present study despite the fact that most previous studies have reported null effects of agreeableness on Internet usage. Given this, there is no empirical or theoretical reason to expect a relationship between agreeableness and Internet usage. However, as the effect of agreeableness on Internet usage has not previously been tested in a Swedish or Nordic context we include it to explore its possible effects. The inclusion of agreeableness is also motivated by the fact that the comparison of our results to previous and future results is made possible (see also Open Science Collaboration 2015 for a discussion about replicability in psychological science).

\section{Conscientiousness}

Conscientiousness is usually described in terms of responsibility, self-discipline, orderliness, competence, and dutifulness. Thus, people who are high in conscientiousness are thorough and efficient, while people low in conscientiousness are careless and lazy (Costa and McCrae 1992). Previous studies have reported mixed findings concerning the relationship between conscientiousness and Internet usage. For instance, Landers and Lounsbury (2006) found that conscientiousness was negatively associated with overall Internet usage, while Mark and Ganzach (2014) found that conscientiousness was positively associated with overall Internet usage. Regarding categories of Internet usage, some studies have found that conscientiousness is positively related to academic activities (e.g., academic research and course participation) and information search (Hughes et al. 2012; Landers and Lounsbury 
2006), while it is negatively related to leisure activities such as listening to music, watching movies, and playing games (Landers and Lounsbury 2006). Mark and Ganzach (2014) found that conscientiousness was positively associated with leisure activities, such as downloading films and music and playing games. Some other studies report that conscientiousness is unrelated to Internet usage (Guadagno et al. 2008; McElroy et al. 2007).

Based on previous research briefly outlined above, we expect conscientiousness to be positively associated with using the Internet for information-based services and duties. Moreover, we expect conscientiousness to be negatively associated with using the Internet for leisure and social activities. People high in conscientiousness will most probably perceive such activities as laziness and irresponsible behavior.

\section{Overall Purpose and Research Questions}

The present study addresses the relationship between the Five Factor Model of Personality and Internet usage. Specifically, this study addresses three research questions. The first question posed is how personality traits are related to overall Internet usage. The second question raised is whether the relationship between personality traits and Internet usage is dependent on the type of Internet activities. The third question posed is whether and how the relationships between personality traits and Internet usage, in terms of overall Internet usage and categories of Internet usage, differ across generation cohorts.

Our study differs from, and as such adds to, previous research in several aspects. First, we use data from a large nationally representative sample. Previous studies on personality traits and Internet usage have not used representative samples. Except for Mark and Ganzach (2014), previous relevant studies have relied on small samples (i.e. $N \leq 220$ ), mostly based on homogenous groups of undergraduate students (e.g., Hamburger and Ben-Artzi 2000). Second, our sample covers a wide range of age groups compared to the sample of Mark and Ganzach (2014), which only included young adults (i.e., 24-28 years of age). Third, our categorization of Internet activities is empirically derived and validated using a nationally representative sample including a wider variety of Internet activities and services. This is in contrast to previous studies, for instance, Hamburger and Ben-Artzi (2000) who proposed a categorization of Internet services based on a very small and homogeneous sample, i.e., 72 students who described themselves as Internet users (82\% from the department of Mathematics) at Bar-IIan University in Israel. Also, their categorization was constructed before the breakthrough of a number of Internet services such as social media, blogging and gaming, indicating that these activities were not included in their list of Internet services.
As previous studies have primarily been based on data from college-aged student samples and young adults, an examination of Internet usage across generation cohorts has to date not been possible. According to Zukin et al. (2006), the Internet is a large-scale project with cultural and economic dimensions which is expected to evoke different behaviors in different generations. Zukin et al. (2006) divided the population into four generation cohorts: DotNets (born between 1977 and 1999), GenXers (born between 1965 and 1976), Baby Boomers (born between 1946 and 1964) and Dutifuls (born between 1910 and 1945). As the younger generations of DotNets and GenXers more or less have grown up with the Internet, and the older Baby Boomers and Dutifuls have learnt how to use digital technology in adult age, differences in digital behavior most probably emerge across generations (Zukin et al. 2006). Based on this reasoning, we anticipate differences in how the personality traits may affect engagement on the Internet across the generation cohorts. This fills an important gap in previous research which has predominantly used either small or age-limited study samples.

\section{Methods}

\section{Sample and Procedure}

The data were collected through a survey study by the SOM Institute. The SOM Institute (the institute for Society, Opinion, and Media) is an independent survey research organization at the University of Gothenburg in Sweden. The institute has since 1986 collected data from the citizens on a wide variety of issues such as political opinion, media use, consumption, occupation, education, health, and well-being.

A postal questionnaire was sent out to 3400 randomly chosen Swedish citizens in the age range of 16 to 85 . The response rate was $53 \%$. Given attrition and missing values, the final sample size of our study was $1694 .^{1}$ Among the 1694 respondents, 762 were males and 932 were females. The mean age was 52.3 years. The data were gathered between September 2014 and February 2015. Each participant received the survey by mail in the middle of September 2014; this was

\footnotetext{
${ }^{1}$ Comparing the composition of our sample with the Swedish population (Statistics Sweden 2018) revealed that the respondents in our sample were overrepresented by Baby Boomers (i.e., $37.1 \%$ in our study sample vs. $28.7 \%$ in the population) and Dutifuls (i.e., $19.9 \%$ in our study sample vs. $14.1 \%$ in the population) and underrepresented by DotNets (23.8\% of the respondents and $36.7 \%$ in the population). GenXers were adequately represented in our sample, i.e., $19.2 \%$ as compared to $20 \%$ in the population. In the group 16-85 years in 2014 (the year of data collection), the mean age in the population was 47 while the mean age of our respondents was 52. In 2014, $50.1 \%$ of the population were females and $49.9 \%$ were males, whereas $55 \%$ of the respondents in our sample were females and $45 \%$ were males. Thus, in the study reported herein younger male citizens were somewhat underrepresented (Vernersdotter 2015).
} 
followed up by seven reminders via telephone and mail up until February 2015 (Vernersdotter 2015).

\section{Measures}

The questions about Internet usage and personality factors analyzed in the present study constituted a small part of the survey.

\section{Personality}

Personality was assessed using the Big Five Inventory, BFI-10 (Rammstedt and John 2007), which is a 10-item inventory with 2 items measuring (some reverse-coded) each personality factor. The BFI has shown satisfactory levels of convergent and discriminant validity as well as test-retest reliability (Rammstedt and John 2007).

Personality trait items were measured using a five-point Likert scale ranging from 1 ("strongly disagree") to 5 ("strongly agree"). The neuroticism index was constructed by averaging the responses to "is relaxed, handles stress well" (reversed) and "gets nervous easily" ( $r=0.40, p<0.01, M=$ $2.57, S D=0.87, N=1613)$. The extraversion index was constructed by averaging the responses to "is reserved" (reversed) and "is outgoing, sociable" $(r=0.46, p<0.01, M=3.58$, $S D=0.91, N=1586$ ). The openness to experience index was constructed by averaging the responses to "has few artistic interests" (reversed) and "has an active imagination" ( $r=$ $0.16, p<0.01, M=3.04, S D=0.94, N=1603)$. The agreeableness index was constructed by averaging the responses to "tend to find fault with others" (reversed) and "is generally trusting" ( $r=0.04, p=0.1, M=3.69, S D=0.68, N=1587)$. The conscientiousness index was constructed by averaging the responses to "tend to be lazy" (reversed) and "does a thorough job" ( $r=0.21, p<0.01, M=4.00, S D=0.75, N=$ 1592). Only respondents who had provided answers on both items to a specific trait were included in the subsequent analyses.

\section{Internet Usage}

Internet usage was operationalized in terms of both overall Internet usage and category-based usage. Overall Internet usage was measured with "How often have you used the Internet during the past twelve months?" to which the respondents were asked to indicate their frequency of usage on a sevenpoint Likert scale, ranging from 1 ("never") to 7 ("daily") $(N=1620, M=6.10, S D=1.84)$. Category-based Internet usage was tapped by "How frequently have you done the following on the Internet during the past twelve months?" The respondents were asked to indicate their level of usage for each of ten different Internet activities or services. Table 1 presents the complete list of activities along with frequency of usage.

Based on the ten Internet activities, categories of Internet usage were submitted to a Principal Component Analysis (PCA) with Varimax rotation. The Kaiser Meyer-Olkin value was .90 and the Bartlett's Test of sphericity was statistically significant $(p<.001)$, supporting the adequacy of conducting a PCA (Bartlett 1954). The selection of the number of factors was guided by Kaiser's criterion (Kaiser 1970). Using this criterion, only factors with an eigenvalue greater than 1.0 were retained for further analyses. Two factors had eigenvalues greater than 1.0, accounting in total for $61.62 \%$ of the variance. ${ }^{2}$ Upon inspection of the verbal contents of the variables, we decided to label Factor 1 "Information and duty activities" and Factor 2 "Leisure and social activities". Table 2 presents factor loadings and the percentage of variance accounted for by each factor.

\section{Demographic Variables and Generation Cohorts}

Age was asked for as an open-ended question. Gender was dummy coded, male was coded as 0 and female as 1 . For generation cohorts we used the four categories of Zukin et al. 2006): (1) DotNets (born between 1977 and 1999), (2) GenXers (born between 1965 and 1976), (3) Baby Boomers (born between 1946 and 1964) and (4) Dutifuls (born between 1910 and 1945).

Age and gender were used as control variables in the analyses of overall and category-based Internet usage. The reason for this was that previous research has shown that age and gender influence Internet usage; men use the Internet more than women and younger people use the Internet more than older people (Hills and Argyle 2003). There are also studies showing that gender moderates the effect of personality traits on the pattern of Internet usage (e.g., Correa et al. 2010; Hamburger and Ben-Artzi 2000). For instance, Hamburger and Ben-Artzi (2000) found that among males, extraversion was positively associated with the use of leisure services and neuroticism was negatively associated with the use of information services on the Internet, whereas for females, extraversion was negatively and neuroticism positively associated with the use of social services on the Internet.

\section{Ethics}

All procedures were in accordance with the ethical standards of the institutional and/or national research committee and with the 1964 Helsinki declaration and its later amendments.

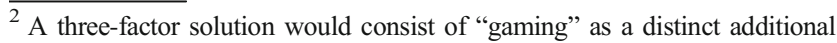
factor, and account for $69.81 \%$ of the variance in the data set. In such a solution, the eigenvalue of the component "gaming" would have been .82 not meeting the Kaiser's criterion.
} 
Table 1 Usage of Internet services in 2014 in the Swedish population

\section{Results}

\section{Internet Usage}

Of the respondents, $9.9 \%$ of the respondents reported that they had not used the Internet at all during the past 12 months, $2.5 \%$ reported that they had used the Internet less than once per week, $5 \%$ on a weekly basis, $11.7 \%$ several times a week, and $71 \%$ on a daily basis.

Table 2 Factor pattern loadings for categories of Internet usage

\begin{tabular}{lrr}
\hline \multirow{2}{*}{ Items } & \multicolumn{2}{l}{ Factors } \\
\cline { 2 - 3 } & 1 & 2 \\
\hline Information and duty activities & & \\
Searching for information/facts & .86 & .25 \\
Sending/receiving email & .86 & .20 \\
Doing banking transactions & .80 & .10 \\
Using news services & .76 & .26 \\
Contacting the authorities & .63 & .31 \\
Buying/ordering goods or services & .62 & .46 \\
Leisure and social activities & & .74 \\
Playing online games & -.03 & .68 \\
Watching movies/TV-series & .32 & .67 \\
Using social media & .37 & .64 \\
Reading blogs & .33 & 11.41 \\
Explained variance (\%) & 50.20 & \\
\hline
\end{tabular}

\section{Personality and Overall Internet Usage}

To examine the influence of the Five Factor Model of Personality on overall Internet usage we conducted a hierarchical multiple regression analysis. Table 3 shows that, after controlling for age and gender, the five personality factors explained a small but significant amount of variance in overall Internet usage (Adj. $\mathrm{R}^{2}=1.2 \%, p<.001$ ) over and above the control variables. Reverse neuroticism (e.g., emotional stability), extraversion and openness to experience were all positively related to Internet usage. No significant associations $(p<.05)$ were found between overall Internet usage and agreeableness or between overall Internet usage and conscientiousness.

\section{Personality and Categories of Internet Usage}

After controlling for age and gender, personality factors explained a significant amount of unique variance in using the Internet for information and duty activities (Adj. $\mathrm{R}^{2}=2.9 \%, p$ $<.001$ ). Reverse neuroticism (e.g., emotional stability), extraversion, openness to experience and conscientiousness were significant predictors of using the Internet for information and duty activities (Table 3 ).

After controlling for age and gender, personality factors explained a significant amount of unique variance (Adj. $\left.\mathrm{R}^{2}=1.7 \%, p<.001\right)$ in using the Internet for leisure and social activities. Openness to experience and reverse conscientiousness were significant predictors of using the Internet for leisure and social activities (Table 3).

In sum, neuroticism and extraversion were, respectively, negatively and positively associated only with using the Internet for information and duty activities. Openness to experience was positively associated with both categories of Internet usage. Interestingly, conscientiousness was positively 
Table 3 Summary of hierarchical regression analyses for the Five Factor Model of Personality predicting overall Internet usage, information and duty activities, and leisure and social activities

\begin{tabular}{llll}
\hline \multirow{4}{*}{ Predictor variables } & \multicolumn{2}{l}{ Outcome variables } \\
\cline { 2 - 4 } & $\begin{array}{l}\text { Overall } \\
\text { Internet usage } \\
(N=1519)\end{array}$ & $\begin{array}{l}\text { Information and } \\
\text { duty activities } \\
(N=1504)\end{array}$ & $\begin{array}{l}\text { Leisure and } \\
\text { social activities } \\
(N=1504)\end{array}$ \\
\hline Step 1 & & & \\
Age & $-.46^{* * *}$ & $-.31^{* * *}$ & $-.57^{* * *}$ \\
Gender & $-.07^{* *}$ & $-.07^{* * *}$ & .01 \\
$R_{\text {Adj }}^{2}$ & $.21^{* * *}$ & $.10^{* * *}$ & $.32^{* * *}$ \\
Step 2 & & & \\
Age & $-.45^{* * *}$ & $-31^{* * *}$ & $-.55^{* * *}$ \\
Gender & $-.07^{* *}$ & $-.08^{* *}$ & .01 \\
FFM of personality & & & .01 \\
Neuroticism & $-.06^{*}$ & $-.09^{* * *}$ & .04 \\
Extraversion & $.07 *$ & $.10^{* * *}$ & $.07^{* * *}$ \\
Openness & $.06^{*}$ & $.06^{*}$ & -.01 \\
Agreeableness & -.01 & -.04 & $-.11^{* * *}$ \\
Conscientiousness & .00 & $.07 *$ & $.34 * * *$ \\
$R_{\text {Adj }}^{2}$ & $.22^{* * *}$ & $.13^{* * *}$ & \\
\hline
\end{tabular}

Entries for predictors are beta weights (i.e., standard regression coefficients)

Abbreviation: FFM Five Factor Model

$*=p<.05 ; * *=p<.01 ; * * *=p<.001$

associated with using the Internet for information and duty activities, while negatively associated with using the Internet for leisure and social activities.

\section{Personality Factors and Internet Usage across Generation Cohorts}

For DotNets (born between 1977 and 1999), personality was not significantly associated with overall Internet usage. However, personality factors were related to the categories of Internet usage. After controlling for age and gender, personality factors explained $3.8 \%$ of the variance in information and duty activities, $\mathrm{F}(7,350)=20.37, p<.001$, and $3.2 \%$ of the variance in leisure and social activities, $\mathrm{F}(7,350)=12.39$, $p<.001$. In this cohort, extraversion was positively related to using the Internet for leisure and social activities. Conscientiousness was the personality factor that best predicted both categories of Internet usage but in opposite directions. Specifically, conscientiousness was positively associated with information and duty activities but negatively associated with leisure and social activities. The standardized regression coefficients from the hierarchical regression analyses for this cohort and other cohorts for overall Internet usage and the two categories of Internet usage are shown in Table 4.

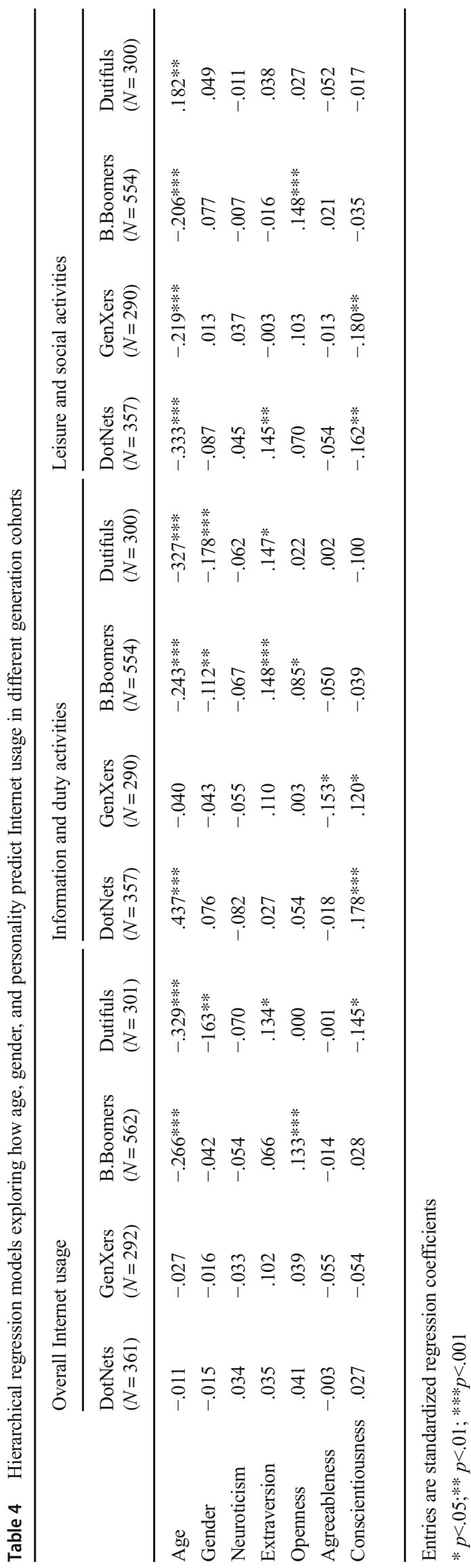


The findings of GenXers (born between 1965 and 1976) were quite similar to the findings of DotNets. Again, no personality factors were significantly associated with overall Internet usage, but two traits (i.e., agreeableness and conscientiousness) were significantly associated with categories of Internet usage. After controlling for age and gender, personality factors did not explain any additional variance in information and duty activities, Adj. $\mathrm{R}^{2}=1.6 \%, \mathrm{~F}(7,283)=1,89$, $p=.071$. In contrast, personality factors accounted for a significant amount of incremental variance in leisure and social activities, Adj. $\mathrm{R}^{2}=2.9 \%, \mathrm{~F}(7,283)=4.18, p<.001$. Similar to DotNets, we found that conscientiousness was positively associated with using the Internet for information and duties but negatively associated with using the Internet for leisure and social activities. We also found that agreeableness was negatively associated with information and duty activities.

For Baby Boomers (born between 1946 and 1964), personality factors explained $2 \%$ of the variance in overall Internet usage, over and above age and gender, $\mathrm{F}(7,555)=$ 9.94, $p<.001$. After controlling for age and gender, personality factors for this cohort explained $2.9 \%$ of the variance in information and duty activities, $\mathrm{F}(7,547)=10.37, p<.001$, and $1.5 \%$ of the variance in leisure and social activities, $\mathrm{F}(7$, $547)=6.39, p<.001$. Among Baby Boomers, extraversion was positively related to using the Internet for information and duty activities. Openness to experience was related to overall Internet usage and both categories of Internet usage, however, it was more strongly related to using the Internet for leisure and social activities.

For Dutifuls (born between 1910 and 1945), personality factors explained $2 \%$ of the variance in overall Internet usage, over and above age and gender, $\mathrm{F}(7,294)=8.76, p<.001$. Overall Internet usage was positively associated with extraversion but negatively associated with conscientiousness. After controlling for gender and age, personality factors for this cohort explained $1.7 \%$ of the variance in information and duty activities, $\mathrm{F}(7,293)=8.66, p<.001$. In contrast, after controlling for gender and age, personality factors did not account for any significant amount of variance in leisure and social activities, Adj. $\mathrm{R}^{2}=1.1 \%, \mathrm{~F}(7,293)=1.90, p=.069$. Similar to the findings of Baby Boomers, extraversion was positively associated with information and duty activities.

\section{Discussion}

Neuroticism was negatively associated with overall Internet usage, whereas extraversion and openness to experience were shown to be positively associated with overall Internet usage. However, exploring these associations across categories of Internet usage and generation cohorts revealed some other interesting patterns which will be discussed in the following.

\section{Neuroticism}

Neuroticism was negatively associated with overall Internet usage and with using the Internet for information and duty activities. This observed association between low levels of neuroticism and more frequent Internet usage went in the opposite direction to some previous research studies (AmichaiHamburger et al. 2002; Amiel and Sargent 2004; Mark and Ganzach 2014). For instance, according to Mark and Ganzach (2014), the Internet provides information that might reduce anxiety for people who are high in neuroticism, such as checking medical symptoms or reading news outlets. The differences between previous and present findings may be due to the characteristics of the study samples. The present study sample had an average age of 52 years while the study sample of Mark and Ganzach (2014) had an average age of 26 years. The differences in observed findings may also be due to the response scale for measuring overall Internet usage. For instance, whereas the response option indicating the most frequent usage of the Internet in the present study was labeled "every day", the response option indicating the most frequent usage of the Internet in Mark and Ganzach (2014) was labeled "several times a day".

Other researchers have also found that neuroticism is positively related to Internet addiction (e.g., Holdoš 2017). Perhaps people high in neuroticism reduce their anxiety through using the Internet several times a day? The negative association between neuroticism and Internet usage was in the present study found only for information and duty activities. This is in line with previous research showing a negative association between neuroticism and using the Internet for learning and educational purposes (Tuten and Bosnjak 2001). A possible explanation, in line with Tuten and Bosnjak (2001), is that information and duty activities primarily have been designed for emotionally stable users. An alternative explanation for why people high in neuroticism use the Internet less frequently for information and duty activities might be that they have another lifestyle than people low in neuroticism, which for instance, might include less frequent contacts with banks and administrative authorities.

\section{Extraversion}

As expected, extraversion was positively associated with overall Internet usage. Moreover, among the two older cohorts, extraversion was positively associated with using the Internet for information and duty activities. In contrast, among younger users, i.e., DotNets, extraversion was positively associated with using the Internet for leisure and social activities.

People who are high in extraversion (e.g., seek external stimulation), use the Internet more frequently. Our result of overall Internet usage and extraversion is consistent with the result of Mark and Ganzach (2014). However, our result is in 
contrast to the negative relationship found by Landers and Lounsbury (2006). One reason for the discrepant findings may be the small sample $(n=117)$ used in the study of Landers and Lounsbury. The number of participants in the present study was 1694 .

Exploring these associations across different generation cohorts helped us to gain a more nuanced understanding. DotNets who scored high in extraversion used the Internet more for leisure and social activities, while Baby Boomers and Dutifuls who scored high in extraversion used the Internet more for information and duty activities. This is in line with a previous study (Correa et al. 2010) which found that the relationship between extraversion and the usage of social media was stronger among younger than older adults. Perhaps Baby Boomers and Dutifuls, who are high in extraversion, seek their leisure and social stimulation from other sources than the Internet? A possible explanation is that social and leisure activities online primarily have been designed for DotNets who are high in extraversion. An alternative explanation might be that DotNets high in extraversion have lifestyles that are suitable for leisure and social activities. Similarly, it might be argued that information- and duty-related services on the Internet have been designed more for Baby Boomers and Dutifuls high in extraversion than for Baby Boomers and Dutifuls low in extraversion. Furthermore, it could be argued that Baby Boomers and Dutifuls high in extraversion have lifestyles that make them engage in information and duty activities to a greater extent than Baby Boomers and Dutifuls low in extraversion. Obviously, more research is needed in this regard.

\section{Openness to Experience}

As expected, openness to experience was positively associated with overall Internet usage in addition to both categories of Internet usage. However, the association between openness to experience and Internet usage was only valid for Baby Boomers.

The finding that openness to experience was positively related to overall Internet usage is in line with most previous research (e.g., Correa et al. 2010; Guadagno et al. 2008; McElroy et al. 2007). However, this finding is not consistent with Mark and Ganzach (2014) who found that openness to experience was unrelated to overall Internet usage. Again, this could potentially be accounted for by the age factor, i.e., the sample of Mark and Ganzach (2014) consisted of only young adults, whereas our sample encompassed several age categories. If we only consider young adults in our sample (e.g., DotNets), our findings are consistent with those of Mark and Ganzach (2014).

Perhaps openness to experience was more related to overall Internet usage in the past, when the Internet was perceived as new and innovative? People who are high in openness to experience are more unconventional. They are more flexible and curious to engage in new experiences. For young adults (e.g., DotNets), in USA and Sweden, the Internet is nowadays perceived as mainstream. For young adults, overall Internet usage is probably not perceived as a new and innovative experience any longer, even if certain activities might be perceived as more innovative. On the other hand, among Baby Boomers, openness to experiences was positively associated with overall Internet usage (and both categories of Internet usage). It is therefore likely that overall Internet usage is perceived as more innovative in this cohort when compared to the younger cohorts. However, following this line of reasoning, we cannot explain why openness to experience is unrelated to overall Internet usage among Dutifuls, the oldest generation cohort.

\section{Agreeableness}

Agreeableness was unrelated to overall Internet usage and to the categories of Internet usage. This result is in agreement with many previous studies showing that agreeableness is unrelated to Internet usage (Kim and Jeong 2015; Mark and Ganzach 2014; McElroy et al. 2007). However, it is worth noticing that we found a negative relationship between agreeableness and information and duty activities for GenXers (Table 4). We do not have any explanation for this specific finding.

\section{Conscientiousness}

We did not find any relationship between conscientiousness and overall Internet usage. However, the analyses of categories of Internet usage revealed some interesting findings. In support of our reasoning, conscientiousness was positively associated with using the Internet for information and duty activities, but negatively associated with leisure and social activities. These relationships between conscientiousness and categories of Internet usage were, however, only valid for the DotNets and GenXers.

A tentative explanation for the reversed relationship between conscientiousness and categories of Internet usage is that people high in conscientiousness prefer structure and planning and may therefore use the Internet more for information- and duty-related services which assist them in organizing their everyday lives. Leisure and social activities might be viewed as more spontaneous and a less structured use of time and therefore more in line with the expected behaviors of people who are low in conscientiousness (Costa and McCrae 1992; Mark and Ganzach, 2014). Our result is in line with Landers and Lounsbury (2006) who found that conscientiousness was positively related to academic activities and negatively related to leisure activities. Our findings also support the hypothesis of Mark and Ganzach (2014) but are not in line with what they found. 


\section{Limitations and Suggestions for Future Research}

The cross-sectional nature of the data only permits us to explore associations and not causal effects. However, it might be argued that personality traits cause the behavior and not the other way around. Another limitation was the self-reporting of Internet usage rather than a measure of real time spent on the Internet and different online activities. In the context of the measurement of Internet usage, it is also worth noticing that the response option indicating the most frequent usage of Internet in the present study was labeled "every day". It is possible that "several times a day" as a response option would have enabled a more fine-grained analysis of the effects of personality traits across the generation cohorts.

Also, personality traits were measured by short scales, which have been shown to be associated with lower reliabilities in the measures (Yarkoni 2010). Caution should also be taken in generalizing the present results to other countries and national cultures. Although the Five Factor Model of Personality has shown universal characteristics, the Internet usage, and the relationship between personality traits and Internet usage, might differ across national cultures. Therefore, we cannot comment on the extent to which present and previous results might have been influenced by national culture characteristics.

Furthermore, the magnitudes of the associations between personality traits and overall Internet usage reported in this study were quite modest, although comparable to previous research results (e.g., Mark and Ganzach 2014). In the present study, we analyzed data from a rather large sample. The larger the sample size is, the smaller the effect that can be detected. That is, when the sample size increases very small effect sizes can become significant, and this is what we see for some of the statistically significant personality trait variables shown in Table 3. However, one should keep in mind that the main reason for larger samples increasing the chance of statistical significance is because they more reliably reflect the size of the effect in the population. Thus, although the effects are small, they may reflect the "true" effects in some sense. Also, it seems reasonable to assume that the modest effects of personality traits on Internet usage may decrease over time as the Internet evolves and for most of us become even more integrated in our everyday lives. Therefore, it is not surprising that we did not find any relationships between personality traits and overall Internet usage among DotNets and GenXers. Moreover, it does not seem farfetched to assume that the effect of personality on overall Internet usage rather will diminish among all users. Thus, we suggest that future research focuses more on categories of Internet usage than on overall Internet usage, and hope that the Internet usage categories and activities used in this study can be useful for future research studies.

Furthermore, as research in this area has been based on survey methodology and correlational evidence, we think that it is time to call for experimental research to examine the causal mechanisms targeting to explain the observed relationships between personality traits and Internet usage for different purposes in different generation cohorts. This would enable a deeper understanding of how personality affects behavior on the Internet and how to design the Internet for different purposes in order to reach out to different generation cohorts with different personal dispositions and preferences.

\section{Conclusions and Implications}

In the present study we used a nationally representative sample to address the relationship between personality and Internet usage by considering overall Internet usage and categories of Internet usage across four generation cohorts.

Our findings indicated that a low degree of neuroticism and high degrees of extraversion and openness to experience were associated with more frequent overall Internet usage. Usage of information- and duty-related services was shown to be negatively related to neuroticism but positively related to extraversion, openness to experience and conscientiousness. On the other hand, usage of leisure and social activities was shown to be positively related to openness to experience but negatively related to conscientiousness. These findings suggest that overall Internet usage is a blunt and insensitive measure as a more nuanced pattern of relationships emerged when categories of Internet usage was employed as the outcome variable.

Furthermore, it was shown that overall Internet usage was only related to personality factors among Baby Boomers and Dutifuls. There were no significant relationships between the personality factors and overall Internet usage among DotNets and GenXers. Apparently, understanding the relationship between personality and Internet usage is not possible without considering the modifying roles of categories of Internet usage and generation cohort.

Future challenges will be to design information- and duty-related services for (1) emotionally unstable users in general; (2) careless and lazy DotNets and GenXers; and (3) introverted Baby Boomers and Dutifuls. Future challenges will also be to design leisure and social activities for (1) introverted DotNets; (2) conscientious DotNets and GenXers; and (3) Baby Boomers low in openness to experience (i.e., cautious and conservative). In the design process, it is also necessary to consider the needs and interests of different users based on their personal dispositions. People low in conscientiousness, for instance, might simply be less interested in services related to information and duty. In other words, it might be a personality issue rather than a design issue. The design challenge is to balance between the unique individual user needs and digital inclusiveness. Therefore, we suggest collaborative efforts between Internet designers and psychologists in order to realize this goal. 
Acknowledgements Thanks are due to the University of Gothenburg, SOM Institute (2016). National SOM 2014. Swedish National Data Service. Version 1.0. https://doi.org/10.5878/002814 for providing access to the data.

\section{Compliance with Ethical Standards}

Ethical Approval All procedures performed in studies involving human participants were in accordance with the ethical standards of the institutional and/or national research committee and with the 1964 Helsinki declaration and its later amendments or comparable ethical standards.

Informed Consent Informed consent was obtained from all individual participants included in the study.

Conflict of Interest The authors declare that there is no conflict of interest.

Open Access This article is distributed under the terms of the Creative Commons Attribution 4.0 International License (http:// creativecommons.org/licenses/by/4.0/), which permits unrestricted use, distribution, and reproduction in any medium, provided you give appropriate credit to the original author(s) and the source, provide a link to the Creative Commons license, and indicate if changes were made.

Publisher's Note Springer Nature remains neutral with regard to jurisdictional claims in published maps and institutional affiliations.

\section{References}

Amichai-Hamburger, Y. (2002). Internet and personality. Computers in Human Behavior, 18(1), 1-10.

Amichai-Hamburger, Y. (2007). Personality, individual differences and Internet use. In A. Johnson, K. McKenna, T. Postmes, \& U. Reips (Eds.), The Oxford handbook of Internet psychology (pp. 187-204). Oxford: Oxford University Press.

Amichai-Hamburger, Y., Wainapel, G., \& Fox, S. (2002). On the Internet no one knows I'm an introvert: Extraversion, neuroticism, and Internet interaction. Cyberpsychology \& Behavior, 5(2), 125-128.

Amiel, T., \& Sargent, S. L. (2004). Individual differences in Internet usage motives. Computers in Human Behavior, 20, 711-726.

Bartlett, M. S. (1954). A note on the multiplying factors for various chi square approximations. Journal of Royal Statistical Society, 16(Series B), 296-298.

Chamorro-Premuzic, T. (2012). Personality and individual differences. West Sussex: BPS Blackwell.

Correa, T., Hinsley, A. W., \& Gil de Zúñiga. (2010). Who interacts on the Web?: The intersection of users' personality and social media use. Computers in Human Behavior, 26(2), 247-253.

Costa, P. T., \& McCrae, R. R. (1992). Revised NEO personality inventory (NEO-PI-R) and NEO five-factor inventory (NEO-FFI): Professional manual. Odessa: Psychological Assessment Resource.

Digman, J. M. (1990). Personality structure: Emergence of the five-factor model. Annual Review of Psychology, 41, 417-440.

Elliott, A., \& Urry, J. (2010). Mobile lives. London: Routledge.

Guadagno, R. E., Bradley, M. O., \& Cassie, A. E. (2008). Who blogs? Personality predictors of blogging. Computers in Human Behavior, 24(5), 1993-2004.
Hamburger, Y. A., \& Ben-Artzi, E. (2000). The relationship between extraversion and neuroticism and the different uses of the Internet. Computers in Human Behavior, 16(4), 441-449.

Hills, P., \& Argyle, M. (2003). Uses of the Internet and their relationships with individual differences in personality. Computers in Human Behavior, 19(1), 59-70.

Holdoš, J. (2017). Type D personality in the prediction of Internet addiction in the young adult population of Slovak Internet users. Current Psychology, 36(4), 861-868.

Hughes, D. J., Rowe, M., Batey, M., \& Lee, A. (2012). A tale of two sites: Twitter vs. Facebook and the personality predictors of social media usage. Computers in Human Behavior, 28, 561-569.

Kaiser, H. (1970). A second generation Little Jiffy. Psychometrika, 35, 173-190.

Kim, Y., \& Jeong, J. S. (2015). Personality predictors for the use of multiple Internet functions. Internet Research, 25(3), 399-415.

Kraut, R., Kiesler, S., Boneva, B., Cummings, J., Helgeson, V., \& Crawford, A. (2002). Internet paradox revisited. Journal of Social Issues, 58(1), 49-74.

Landers, R. N., \& Lounsbury, J. W. (2006). An investigation of big five and narrow personality traits in relation to Internet usage. Computers in Human Behavior, 22(2), 283-293.

Mark, G., \& Ganzach. (2014). Personality and Internet usage: A largescale representative study of young adults. Computers in Human Behavior, 36, 274-281.

McElroy, J. C., Hendrickson, A. R., Townsend, A. M., \& DeMarie, S. M. (2007). Dispositional factors in Internet use: Personality versus cognitive style. MIS Quarterly, 31(4), 809-820.

Open Science Collaboration. (2015). Estimating the reproducibility of psychological science. Science, 349, aac4716.

Persson, M., \& Eriksson-Björling, M. (2011). Mobiltelefonen Förändrade kommunikationsvanor och ökade kostnader. In J. M. Roos (Ed.), Konsumtionsrapporten (pp. 31-34). University of Gothenburg: Centre for Consumer Research.

Rammstedt, B., \& John, O. P. (2007). Measuring personality in one minute or less: A 10-items short version of the big five inventory in English and German. Journal of Research in Personality, 41(1), 203-212.

Soto, C. J., John, O. P., Gosling, S. D., \& Potter, J. (2011). Age differences in personality traits from 10 to 65 : Big five domains and facets in a large cross-sectional sample. Journal of Personality and Social Psychology, 100(2), 330-348.

Statistics Sweden. (2018). Population by age and sex. Year, 1860-2016.

Swickert, R. J., Hittner, J. B., Harris, J. L., \& Herring, J. A. (2002). Relationship among Internet use, personality and social support. Computers in Human Behavior, 18(4), 437-451.

Tuten, T., \& Bosnjak, M. (2001). Understanding differences in web usage: The role of need for cognition and the five factor model of personality. Social Behavior and Personality, 29(4), 391-398.

Vernersdotter, F. (2015). Den nationella SOM-undersökningen. In A. Bergström, B. Johansson, H. Oscarsson, \& M. Oskarson (Eds.), Fragment (pp. 563-588). University of Gothenburg: The SOM-institute.

Yarkoni, T. (2010). The abbreviation of personality, or how to measure 200 personality scales with 200 items. Journal of Research in Personality, 44(2), 180-198.

Zukin, C., Keeter, S., Andolina, M., Jenkins, K., \& Carpini, M. X. D. (2006). A new engagement?: Political participation, civic life, and the changing American citizen. Oxford: Oxford University Press. 